\section{DIAGNOSTICO Y RETOS DEL ECONOMISTA EN EL PERÚ}

Dra. BEATRIZ HERRERA GARCÍA

XIII CONFERENCIA DE AFEIEAL Santo Domingo, República

Dominicana, UASD

\section{DIAGNÓSTICO Y RETOS DE LA ECONOMÍA LATINOAMERICANA Y EL CARIBE}

\section{RESUMEN}

Un prestigioso investigador de nuestros tiempos, Peter Drucker, ha publicado varias obras en la que presenta el resultado de sus investigaciones, en torno a situaciones de investigación y desarrollo (I \& D). En ese sentido, afirma que el crecimiento del producto y el requerimiento de la labor del obrero fabril, del agricultor tradicional y, en general, del trabajador poco calificado, capacitado y muy repetitivo, se están moviendo en sentido opuesto porque los requerimientos de la fuerza laboral están cambiando rápidamente. E.g.. En la década de los treinta, dos tercios de la población de los Estados Unidos realizaban labores eminentemente manuales, en la presente década dichas actividades abarcan apenas el veinte por ciento de su población, situación que se repite en países del área desarrollada y en algunos países subdesarrollados.

Los grandes cambios mundiales y tecnológicos datan de los años setenta. estos cambios han provocado fenómenos estructurales que se explican por las nuevas tecnologías, la automatización de los procesos y el aumento de la eficiencia y la productividad del trabajo. Y sobre todo por la primacía que está alcanzando el conocimiento, el saber como factor de producción.

Según el mismo autor, profundas transformaciones se están dando en el mundo laboral. Es decir, el operario repetitivo, poco calificado y sin iniciativa, se va quedando progresivamente sin opciones de conseguir nuevos empleos. Hacia el siglo XXI no habrá ningún país desarrollado en el que los obreros tradicionales, los que fabrican y trasladan mercancías, representen más de una sexta u octava parte de la fuerza laboral total. Porque el centro de la economía se va desplazando hacia las actividades de producción y distribución del conocimiento.

La desmaterialización del producto, resultado de los grandes cambios tecnológicos y del nuevo papel de la industria, se expresa en un clásico ejemplo de la confección de una camisa de algodón; las modernas camisas contienen en la actualidad poco valor material agregado o añadido, así el valor del hilado de algodón que se utiliza para su confección (incluyendo el valor del algodón. la mano de obra y desgaste de maquinarias e instalaciones) no llega a superar en el mejor de los casos el 10 por ciento del valor total de la camisa, la diferencia corresponde a la innovación, a la creatividad, a la incorporación del nuevo conocimiento. "Antaño los que trabajaban en la industria tenían ampollas en las manos, ahora en cambio. usan el cerebro".

Ya no es posible ser competitivo en base a trabajo intensivo o extensivo; es más, en el mundo actual no se puede competir en base a salarios bajos y mano de obra abundante y poco calificada. 
En las organizaciones empresariales modernas y competitivas de cara al mercado mundial. los trabajadores requieren un gran bagaje de conocimientos especializados. La población. toda, debe ser mejor instruida y ser culta para que pueda aplicar su conocimiento en proceso productivo y de servicios, los hombres y mujeres de hoy y el futuro deben ser capaces de comprender la realidad en permanente cambio y transformación y, de desarrollo innovador constante.

El conocimiento, producto de la formación superior y de la especialización. a veces no puede ser aplicado por falta de una cultura productiva y transformadora, quedándose en consecuencia en sólo información, es decir. en pocas oportunidades se convierte en fuente de producción, por ello la aplicación del conocimiento consiste en aumentar su productividad, su rendimiento. Debe siempre manifestarse a través de su efecto multiplicador, propagador, de la puesta en práctica de todo lo que sabemos y hemos aprendido a través del estudio. Se trata de relacionar el conocimiento con su aplicación concreta a nuevos productos y servicios.

La productividad del conocimiento es un factor importante de la competitividad de una empresa o de un país: es también. un gran desafío que pone en primer lugar el tema de la importancia de la educación superior especializada y de su rol en la transformación de la economía y sociedad.

\section{LA FORMACIÓN PROFESIONAL UNIVERSITARIA. ETAPAS Y PERÍODOS}

\section{La profesionalización en la década de los sesenta.}

El sistema universitario peruano. desde su organización y concepción. ha estado históricamente desvinculada de los problemas de la producción doméstica y de los niveles de subdesarrollo imperantes. La empresa o las organizaciones empresariales, no han tenido ni han desarrollado vínculos con las academias, porque el desarrollo nacional no fue el destino de la clase dirigente. La mayor o abrupta abundancia de recursos naturales hizo que su inserción en el contexto internacional y su forma de explotación, tuvieran un carácter primario; en ese sentido, la economía se dinamizó de acuerdo a las tendencias de los sectores extractivos de alta rentabilidad internacional, la demanda externa fue un factor determinante que orientó el destino de la producción doméstica.

Un ejemplo de la inexistencia de un interés real por una verdadera industrialización, es el hecho de que sólo en la década de los sesenta se llega a promulgar la ley del comercio y la ley de industrias. Nunca antes se consideró que la transformación de los productos primarios fuese base para el desarrollo nacional, este llegaría según unos a través del mercado internacional, cuyos precios favorecían el acopio de divisas en forma creciente. asegurando de esa manera el financiamiento de todas las demás necesidades de la población y de la economía doméstica.

La sustitución de importaciones, forma que adquirió la industrialización entre las décadas cincuenta y sesenta, fue diseñada con una estrategia favorable a las empresas transnacionales porque ésta no les generaba competencia en los mercados domésticos. La industrialización en esencia, era un sistema de ensamblaje de piezas y partes desarrolladas y fabricadas en el exterior, era una sustitución industrial de productos de bienes finales de consumo duradero, nunca se diseñó una industria que se basara en los recursos nacionales disponibles y que trans- 
formara las materias primas en productos de mayor valor agregado: en ese sentido fue una industria consumidora de divisas que no generaba. éstas provenían de las exportaciones de los sectores primarios.

Es decir, estos sectores eran los grandes financiadores del proceso industrial. que irradiaba mayor dependencia del exterior en detrimento de un proceso de integración nacional; funcionó dentro de un círculo vicioso con efectos perversos y contrarios a los sectores financiadores, pues éstos últimos fueron mermando cada vez más su capacidad acumulativa, fueron por esa vía siendo descapitalizados progresivamente.

Dentro de este tipo de relaciones. la educación en general y la universidad en particular, siguieron patrones de desarrollo mimetizados, en el caso de las universidades el modelo de desarrollo fue y sigue siendo la universidad "bonapartista"; es decir, una universidad formadora de profesionales generalizantes, apoyados en una formación memorística, repetitiva y poco crítica. La ciencia y la tecnología, la investigación y el desarrollo científico, no fueron tomados en cuenta como sustento y esencia de la existencia universitaria. Esos aspectos, al igual que la industrialización debían ser generados y desarrollados estratégicamente por los centros. correspondiéndonos sólo su aplicación de acuerdo a las realidades nacionales, intrínsecas y propias de los procesos en concreto.

El desarrollo universitario de esa época correspondió a los líderes intelectuales, compuestos por letrados, jurisprudentes, educadores, filósofos y contadores mercantiles. La formación profesional universitaria tenía esas connotaciones ideológicas. se orientaba hacia la universalización y generalización de las ciencias y las letras, el sentido humanístico primaba al sentido productivista. El desarrollo era un proceso espontáneo y dependía de la mayor y abundante dotación de factores naturales; el crecimiento no parecía tener límites y los profesionales podían desenvolverse en cualquier campo con una eficiencia promedio aceptable. porque el proceso de industrialización estaba en ciernes y era totalmente dependiente del exterior.

\section{La intervención del Estado y los cambios en la formación profesio- nal.}

En los albores de los años setenta se produce un importante fenómeno en el proceso político peruano, producto de la crisis y el entreguismo. Los militares como institución, asumen el gobierno del país en un modelo de revolución participativa: es decir, en un marco de reformas sustanciales en las que el actor y sujeto fundamental vendría a ser el estado. El rol del estado en el desarrollo y la liberalización nacional, se traducía en la ingerencia del estado en la marcha del país, en las diversas instituciones y en la organización empresarial que asume nuevos retos a partir de una empresa pública de interés social. Es la reorganización y reestructuración del sistema económico peruano mediante la planificación estratégica dirigida por los militares, para superar el subdesarrollo económico y el atraso social de las mayorías nacionales.

En esas circunstancias el estado sufre profundos cambios y los diversos ministerios se reorganizan en función de dirigir y orientar el desarrollo a través del rol fundamental del estado. en la dinámica económica y social. La inversión y el empleo son fuertemente estimulados por el gasto social. la inversión pública y las reformas estructurales.

Las nacionalizaciones, las estatizaciones y las expropiaciones están a la orden 
del día retando al capital privado nacional y extranjero a emular con el estado. Sin embargo, éstas no corresponden al esfuerzo y entusiasmo de los militares. Decae la inversión privada en general y el estado se involucra en cubrir esos vacíos con mayor gasto presupuestal y con nuevos préstamos internacionales. Al inicio la revolución despertaba simpatías en el campo internacional, por lo que su acceso al financiamiento internacional fue favorecido, asimismo, la enorme liquidez internacional jugó a favor del fácil y rápido endeudamiento del país.

El modelo de desarrollo. propiciaba un gran proceso de integración nacional y regional a partir de dos premisas: el proceso de industrialización y la reforma agraria, en ese esquema la formación profesional asume nuevos retos; ante los nuevos escenarios la universidad peruana sufre grandes y contrastantes cambios, la continuidad es interrumpida cual punto de inflexión, y se inicia una reforma universitaria que violenta los principios democráticos. la autonomía de su organización y gestión y, castra su esencia. al trasladar la investigación y la formación de postgrado a otras instituciones.

La universidad peruana se mimetiza al modelo norteamericano, se imponen los estudios generales, se liquidan las facultades y se instauran los departamentos y programas académicos. Es una estructura orientada a lograr una mayor productividad profesional, se requieren profesionales para impulsar y dirigir el proceso de reformas. Se diluye la formación superior, se crean instancias, otros niveles como las ESEP: se privilegia la formación politécnica y de mandos medios con una gran improvisación.

El sistema departamentalista, que agrupa a profesionales de una misma rama y. el sistema de estudios generales define una estructura curricular flexibie. de créditos obligatorios y electivos que individualiza la formación profesional por un lado, y destruye la organización existente hasta entonces por otro. En este período se produce un divorcio en la relación y nexo entre los profesionales y las especializaciones, cada grupo profesional se encierra corporativamente. defiende sus fueros y delimita sus campos. Los colegios profesionales se fortalecen e inician una aguda campaña de diferenciación tratando de ser más competitivos.

Es un período rico en contradicciones, los postgrados -maestrías y doctorados-son postergados, porque el nuevo modelo universitario es considerado extremadamente elitista, por lo que corresponde sean otras instituciones y el extranjero los que desarrollen esa función. Así, el perfil profesional sufre ajustes importantes, el sentido humanístico es sustituido por la ideología productivista y la formación especializada.

\section{Las facultades del nuevo tipo y los cambios en la década de los ochenta.}

La década de los ochenta es la década de las frustraciones. Agobiados por la crisis de la deuda externa, el país enfrenta nuevos retos; la industrialización nuevamente se frustraba por los "embotellamientos o estrangulamiento externos", además el conjunto de reformas impulsadas por el gobiemo militar habían entrado en un proceso de fracaso por falta de financiamiento e inspiración. El Estado era incapaz de redirigir la dinámica económica; nuevos gobiernos de origen democrático enfrentarían los nuevos retos y desandarían el proceso de reformas propulsados por el estado. La situación en general, es altamente conflictiva por la dimensión de la crisis y la escasez generalizada de financiamiento externoen sus dos modalidades: IDE y créditos o préstamos. 
El sistema universitario peruano había madurado y estaba en condiciones de reorganizarse, las experiencias de los setenta fueron asimiladas críticamente; por un lado, el divorcio $y / 0$ separación entre departamentos y programas tenía que acabar y por otro lado, los estudios generales no habían dado resultados y amenazaban con prolongar la formación profesional universitaria. El currículum y el perfil de las carıeras requerían ajustes de concepción y contenido, en ese sentido debían ser el resultado del esfuerzo de colectivos de docentes polivalentes; es decir, la departamentalización podía ser provechosa si operaba de manera conjunta dentro de un objetivo curricular común; para ello los grupos de docentes agrupados por especialidades debían estar estrechamente relacionados, interactuando en un proyecto de formación profesional específico.

Era necesaria una nueva ley universitaria que reorganizara el funcionamiento y la concepción de las universidades a partir del concepto y la importancia de las facultades. El retorno de las facultades fue un proceso de desarrollo en la universidad, porque no fue un retorno a las facultades señoriales que con la cátedra permanente y apropiada por los profesores principales impedía el avance académico y frenaba el desarrollo del pensamiento crítico. En las nuevas facultades o facultades de nuevo tipo se abren nuevas posibilidades al pensamiento universitario a través de la cátedra paralela y, del acceso a la misma de profesores de todas las categorías. Así, se creaban las condiciones para la investigación para toda la comunidad universitaria, en sus dos niveles la aplicada y la teórica.

En este período de crisis y como en toda crisis surgen los elementos del nuevo sistema, surgen nuevas fuerzas que desarrollan la ciencia y la sociedad; uno de esos logros fueron las facultades de nuevo tipo. or- ganizadas en torno a proyectos formativos de profesionales más competitivos. Eso sucedió en los campos de las ciencias, las ingenierías y las letras; e.g., la antigua Facultad de Humanidades ya no respondía al momento; los requerimientos profesionales eran más específicos y exigentes, ahora eran necesarias facultades como letras; ciencias sociales; educación; comunicación, sicología. etc. En las nuevas facultades los docentes de las diversas especialidades fueron integrados, cada uno de ellos era responsable de aportar proporcionalmente a la formación de un profesional determinado. Resurgieron las escuelas de pregrado y las de postgrado, en una secuencia de continuidad en la preparación y formación académica de profesionales más capacitados y competitivos. El pregrado cumple con objetivos de formación integral, en cambio el postgrado cumple con objetivos diferentes, de especialización. de mayor dominio académico y de aplicación concreta en las diversas ramas profesionales.

Este nuevo concepto de estructura universitaria funde en la facultad, la vida y el desarrollo universitario, pone al servicio de la formación profesional todas sus unidades: la escuela, el postgrado, los centros e institutos de investigación, los departamentos académicos, CEUPS (Centro de extensión universitaria y proyección social), las prácticas profesionales, etc. Adicionalmente, para el desarrollo científico y académico se crean las maestrías y los programas doctorales para los más destacados, ambas están restringidas por sus exigencias y por su orientación que difieren en gran parte a los objetivos de profesionalización.

Ahora los profesionales que forman las universidades del sistema peruano, se ajustan más a las tendencias de la modernización del país y están estrechamente vinculadas a las tendencias internacionales. La ciencia y la tecnología, 
operan como una unidad y se traducen en el lenguaje universitario en profesionales competitivos.

\section{NUEVOS ESCENARIOS, NUEVOS ENFOQUES}

\section{El papel de las investigaciones en la continuidad del desarrollo profesional en el umbral del Siglo XXI.}

El desempeño profesional es cada vez más exigente, más competitivo en todas las ramas profesionales. Este obedece al resultado de un gran proceso de cambio y desarrollo mundiales: así a partir de los setenta. en el mundo industrializado se crean las condiciones para el surgimiento de lo que se ha dado en llamar la "tercera revolución industrial". Los nuevos adelantos científicos y tecnológicos han creado nuevas bases del desarrollo universal y, están transformando rápidamente la estructura y concepción de la actividad manufacturera, por una creativa, innovadora en lugar de la agregación simple de valores materiales; es decir, por la incorporación ilimitada del conocimiento en el proceso productivo, por la desmaterialización del producto. También, la importancia y vitalidad del sector o actividades secundarias, están siendo reevaloradas por el papel dinámico del sector $o$ actividades terciarias o de servicios.

Son tiempos de cambio, de transformación y en el centro de ello se sitúa al conocimiento como motor de esos procesos. La modernización del mundo parte del nuevo concepto del papel y el rol de la educación; hoy en día, la inversión más rentable y más productiva es la inversión en el hombre, en su educación y cultura. Los sistemas económicos serán más armónicos y mejorarán las condiciones de su inserción internacional si en el corto y mediano plazo dan grandes saltos en materia de educación. Un ejemplo de ello lo dan los Países Asiáticos de reciente industrialización, su presencia mundial y su importancia, son debidos al gran esfuerzo que realizaron en el pasado inmediato en materia de inversión en educación, sus éxitos son gracias a la aplicación de las innovaciones en todos sus procesos.

Invertir en educación es preparar más y mejor, es comprometer en los programas educativos al mayor número de jóvenes en edad escolar para prepararlos en distintos niveles en las artes y las ciencias, en el dominio de las tecnologías y de su aplicación profesional. En hacer del conocimiento una aplicación práctica rutinaria, con el fin de transformar las cosas, de cambiarlas para producir mejores resultados: en hacer de la inventiva una actividad rutinaria.

¿Cómo la universidad puede cumplir con ese objetivo? La estructura de la universidad es permisible al cambio y al desarrollo, esto se puede observar cuando sus capacidades en materia de formación profesional son combinadas con su capacidad investigativa. El desarrollo académico es un proceso que se da a partir de las investigaciones teóricas y aplicadas, luego ambas son aplicadas en los programas de formación profesional; en ese sentido, el aprendizaje y dominio de las ciencias son un desarrollo continuo que se inicia con el proceso de admisión vía selección de los mejores, continúa en la formación de pregrado $\mathrm{y}$, posteriormente, sigue en la capacitación de los postgrados. Es un largo camino de relación estrecha con la ciencia y su aplicación práctica a los procesos concretos, es una manera nueva de pensar y aplicar los conocimientos.

\section{Las nuevas exigencias del ejercicio profesional en el siglo XXI.}

Los profesionales para ser más efi- 
cientes y competitivos deben mantenerse en reciclaje permanente, el ejercicio de las profesiones exige estar al día con los nuevos inventos y descubrimientos que se dan en su ramo, no sólo basta estar informado de ello sino fundamentalmente de estar capacitado para poder aplicarlo en su "Iutina" diaria. para hacer de su trabajo una actividad interesante, creativa y conducente a una mayor productividad. Estos objetivos del presente, serán más urgentes e intensivos en el próximo siglo; porque además, las tendencias del conocimiento y de la formación profesional especializada es a integrarse más en una nueva dimensión, e.g., en las ciencias sociales ahora, debido a la globalización financiera y a la importancia de las finanzas dentro de su profesión, requiere de conocimientos especializados de las ciencias administrativas. de las ciencias económicas, de las ciencias de la información. de la computación y de las ciencias financieras; todo ello redundará en un mejor ejercicio profesional y un mayor rendimiento.

Reciclaje de los conocimientos es la clave, los nuevos desafíos científicos y sus formas de aplicación en las profesiones resoectivas es el sentido y el contenido de a segunda especialización. Los profesionales no pueden adecuarse a los nuevos tiempos con sus conocimientos viejos, están obligados a no bajar la guardia en su preparaión, esta debe ser una tarea continua, estuJıar, investigar, descubrir y aplicar, debe ser una constante en cada uno de nosotros y, el medio más adecuado para ello es la segunda especialización; involucrarse de manera permanente en estos programas universitarios es y será una condición para el ejercicio profesional eficiente. En dichos programas universitarios los profesionales de un mismo ramo se reúnen para discutir académicamente los alcances de la profesión para darle un contenido científico a sus experiencias personales y, para comparar y encarar de manera conjunta soluciones creativas a temas concretos. La necesidad de la vida colecti$v a$, del trabajo en equipo con sus pares profesionales le da nuevas dimensiones y horizontes al desempeño profesional. La necesidad de especializarse permanentemente es una condición necesaria y suficiente para la vida profesional. La organización de los colegios profesionales y los profesionales por colectivos de especialista le confiere nueva vida a partir de las nuevas necesidades profesionales; por lo que resulta inevitable una estrecha vinculación entre los colegios profesionales y los programas universitarios de segunda especialización. No sólo para elevar el nivel de los miembros de la orden; sino fundamentalmente. para incorporar la ciencia al ejercicio profesional permanente.

\section{El rol de las investigaciones económicas y sus exigencias en la actualidad y el futuro.}

Todo lo anterior se enmarca dentro de un escenario cambiante. donde la complejidad del funcionamiento de las organizaciones y cada vez más creciente internacionalización comercial y financiera, hacen evidente la necesidad de contar con personal altamente calificado, capaz de enfrentar los retos del mercado de trabajo en áreas de conocimiento que requieren de una sólida preparación académica y con dominio y técnicas que le permitan resolver nuevas situaciones producto del desarrollo del cambio. En este sentido, la adecuación de los estudios y preparación de la nueva universidad, resulta indispensable para dar respuesta no sólo al nuevo orden económico internacional, sino también, a las necesidades científicas y tecnológicas que requieren ser satisfechas en las organizaciones.

El siglo $X X$ en que vivimos se ha caracterizado, entre otros aspectos, por una marcada importancia de la economía, enfatizada en la década de los noventa, al 
pasar de una economía vinculada principalmente con la guerra armamentista (conflicto Este-Oeste), a la integración de bloques económicos que crean estrategias financieras para el mejor aprovechamiento de los recursos con que cuenta cada país. El movimiento financiero internacional a medida que se expande, se forma más complejo e interdependiente, repercutiendo en las economías nacionales en los sectores público y privado.

En el marco gubernamental, la eficiente administración pública y financiera es decisiva para el bienestar de la comunidad. mediante la prestación de servicios públicos, apertura de fuentes de trabajo y financiamiento en materia de vivienda y de seguridad social. entre otros. El gobierno garantiza la estabilidad económica y vela para que la economía y las finanzas de toda la nación. aseguren el incremento del desarrollo económico y social de todos los sectores, estableciendo prioridades que permitan resolver la necesidad de una mejor distribución de la riqueza, mediante la obtención y aplicación racional de sus recursos financieros. La apertura económica propicia que las empresas compitan por capitales y mercados internacionales, dando lugar a nuevas estructuras financieras, cuya complejidad dificulta el manejo de las empresas que generan un gran volumen de operaciones y cuya rotación de recursos monetarios deberá ser equilibrada y eficaz, a fin de dar por resultado una empresa progresista con rentabilidad y solvencia; lo cual no sólo influye en todas las áreas de la organización, sino también en el bienestar del personal que la integra.

Por otro lado, el entorno social y económico en que se desenvuelven las empresas, presenta constantes cambios que repercuten en su funcionamiento. Entre las modificaciones económicas que se han dado en el país en la presente década, cabe destacar; la disminución de la tasa de la inflación, así como de la paulatina caída de la tasa de interés, deslizamiento de la moneda, un retorno a la productividad como elemento trascendente para el desarrollo, las distintas formas de financiamiento; el mercado de valores que inicia una apertura y constituye una opción adicional de inversión $y$ el hecho de que muchas empresas se acercan a fuentes de financiamiento $y$ mercados externos; dando lugar a la internacionalización comercial, sujeta a reglas internacionales.

Durante los procesos de globalización las empresas compiten, principalmente, por capitales, mercados y tecnología; lo cual origina que la utilización de los estados financieros deje de ser meramente local, para tener un aprovechamiento externo por inversionistas extranjeros. También se observan constantes cambios en la política fiscal que inciden en los impuestos, así como, una transformación acelerada en los sistemas de información que cada día se tornan más sofisticados.

La dinámica del cambio en que se encuentra inmerso el país, hace necesario preparar individuos que se conviertan en agentes que propicien el cambio y las transformaciones en las mejores condiciones posibles. El desarrollo de la nueva universidad permitirá a las empresas contar con personal calificado y capaz de dar respuesta a las nuevas necesidades que plantea el nuevo orden económico nacional e internacional, mediante la aplicación de sus conocimientos al campo concreto del ejercicio profesional, así como de la investigación para proponer soluciones originales y creativas, viables a las empresas de diferentes países.

Toda la problemática indicada pone de relieve la importancia de la investigación científica; conlleva la idea de formar 
nvestigadores, porque resulta inconcebibles programas de postgrados sin un papel relevante de la investigación como parte medular de esos estudios. Así, los programas de doctorado, en términos generales, se enfocan a la formación de individuos creativos y capaces de producir conocimiento original y desarrollar el existente. Uno de los medios más adecuados para lograr este objetivo, es mediante el desarrollo de los seminarios. Los seminarios de investigación deben enfocarse a la guía de la investigación y a la adquisición de técnicas y conocimientos, así como de inducir al crecimiento de la capacidad creadora y hacerla fructífica. Al interior de los seminarios deben tener lugar el análisis, la crítica y la autocrítica, la búsqueda conjunta de soluciones tendientes a fortalecer el aprovechamiento y rendimiento de los participantes.

\section{CONCLUSIONES}

1.- Los objetivos y la orientación de la formación profesional universitaria ha ido cambiando paulatinamente, de acuerdo al desenvolvimiento del proceso histórico peruano. A medida que la modernización de los sistemas era el objetivo de las políticas, los perfiles profesionales y los currículos correspondientes se ajustaban.

2.- Sin embargo, en los nuevos tiempos han surgido nuevas exigencias derivadas del rápido desarrollo científico y tecnológico, que de manera abrupta está modificando las estructuras sociales, económicas, políticas y militares. La formación profesional universitaria ha pasado por varias etapas, de la formación genérica y humanista, a la formación profesional integral y luego a la formación profesional especializada. Ahora, en un nivel cualitativamente superior, requiere de conocimientos integradores hacia las ciencias afines para mejorar y hacer más eficiente su perfil y ejercicio profesional. En un sistema de educación superior continuada, esto puede combinarse y lograrse a través de la implementación e importancia de los Programas Universitarios de Segunda Especialización.

3.- Las universidades han sido reformadas y reestructuradas por diversas leyes universitarias. En la actualidad, las universidades se desarrollan entorno a las Facultades de Nuevo Tipo, que como resultado de las experiencias anteriores, han recogido lo mejor de los anteriores sistemas; por un lado, la organización insuperable para la formación profesional a través de las facultades y, por otro. la participación de docentes altamente especializados y estrechamente vinculados, provenientes del sistema departamentalista. Las nuevas facultades están dotadas para desarrollar la vida académica mediante la investigación, es decir, para asimilar y desarrollar la ciencia aplicándolas en las formaciones profesionales específicas. El sentido real de la educación continuada puede traducirse en el esfuerzo universitario en cuestiones de capacitación y especialización, en el reconocimiento de la importancia, el papel y el rol de las segundas especialidades.

4.- Al ser el conocimiento el factor fundamental de transformación y desarrollo, las segundas especializaciones pueden asegurar de manera continuada cómo los profesionales pueden estar no sólo permanentemente actualizados; sino hacer de los profesionales, hombres de ciencia a partir de sus experiencias y del tratamiento científico de los problemas concretos. Además, de vincular y acercar, en función de un objetivo común cómo es la excelencia profesional, a las universidades (a través de sus segundas especializaciones) con los colegios profesionales revitalizados por el surgimiento de colectivos de miembros de la orden agrupados por especialidades desarrolladas en los programas de segunda 
especialización.

\section{BIBLIOGRAFÍA}

CASTRO S., Pedro

1997. Historia financiera del Perú: 1960 -

1990. Tomo II , USMP, Lima.

CASTRO S., Pedro

1992. Teorías del desarrollo, crítica a la teoría de la Cepal, UNMSM, Lima.

DE SOTO, Hernando

1986. El Otro Sendero. Instituto Libertad y Democracia. Lima.

FIGUEROA. Adolfo

1995. En: La Cuestión Distributiva en el

Perú. En: Instituto de Estudios Peruanos

FORGUES, Roland

1993. Peru, Entre el desafío de la violencia y el sueño de lo posible. Lima .

GONZALES DE OLARTE. Efraín

1995. En: Transformación sin desarrollo: Perú 1964-1994. IEP, Lima.

INSTITUTO DE FOMENTO DE UNA EDUCACIÓN DE CALIDAD

1991. Política educativa y política social en la estrategia del de sarrollo, Lima.

MALPICA, Carlos.

1989. El Poder económico en el Perú, Lima.

ROCHA V., Alberto

1988. La Militarización del Estado, América Latina 1960-1980, Lima.

THORP. Rosemary / BERTRAM, Geoffrey 1985. Perú: 1890-1997. Crecimiento y políticas en una economía abierta. Lima.

WEBB. Richard / Figueroa, Adolfo

1975. Distribución del Ingreso en el Perú, Lima. 